\title{
CORRIGENDUM
}

\section{Increased estimates of air-pollution emissions from Brazilian sugar-cane ethanol}

C-C. Tsao, J . E. Campbell, M. Mena-Carrasco, S. N. Spak, G. R. Carmichael and Y. Chen

Nature Climate Change 2, 53-57 (2012); published online 11 December 2011; corrected online 6 March 2012.

In the version of this Letter originally published, the affiliation for M. Mena-Carrasco should have read: Department of Environmental Engineering, Universidad Andres Bello, Santiago, 8370251, Chile. This error has been corrected in the HTML and PDF versions of the Letter. 